\title{
Inelastic diffraction and color-singlet gluon-clusters in high-energy hadron-hadron and lepton-hadron collisions
}

\author{
Meng Ta-chung, R. Rittel and Zhang Yang \\ Institut für Theoretische Physik, Freie Universität Berlin, 14195 Berlin, Germany \\ e-mail: meng@physik.fu-berlin.de; rittel@physik.fu-berlin.de; zhangy@itp.ac.cn
}

\begin{abstract}
It is proposed, that "the colorless objects" which manifest themselves in largerapidity-gap events are color-singlet gluon-clusters due to self-organized criticality (SOC), and that optical-geometrical concepts and methods are useful in examing the space-time properties of such objects. A simple analytical expression for the $t$-dependence of the inelastic single diffractive cross section $d \sigma / d t$ ( $t$ is the fourmomentum transfer squared) is derived. Comparison with the existing data and predictions for future experiments are presented. The main differences and similarities between the SOC-approach and the "Partons in the Pomeron (Pomeron and Reggeon)"-approach are discussed.
\end{abstract}


Diffraction in Optics can be used as an instrument to determine the unknown wavelengths of incident waves from the known geometrical structures of scatterers and vice versa. This has been pointed out and demonstrated by von Laue and his collaborators in their celebrated papert eighty-five years ago. Based on this idea, a series of experiment 2 have been performed in the 1950's and 1960's to measure the sizes of various nuclei by using hadron-beams (as hadronic waves) where particle-accelerators have been used as "super microscopes" Theoretically, the idea of using optical and/or geometrical analogies to describe high-energy hadron-nucleus and hadron-hadron collisions at small scattering angles has been discussed by many authors many years ago. It is shown In $^{\text {in }}$ particular that this approach is very useful in describing hadron-hadron elastic scattering.

Since the recent observation I of large-rapidity-gap (LRG) events in deep-inelastic electron-proton scattering in the small- $x_{B}$ region $\left(x_{B}<10^{-2}\right.$, say) - a kinematical region where soft gluons dominate 6 , much attention l $^{6}$ has been attracted by inelastic diffractive scattering processes in lepton- and hadron-induced high-energy collisions terest in this kind of events is mainly due to the fact that the occurrence of LRG's strongly suggests the existence of certain "colorless object(s)", the "exchange" of which plays a dominating role in such processes. The "colorless object" which carries the quantum-numbers of vacuum has been given䀦 13 different names (Pomeron, Reggeon etc.), and it has been suggested 2 . 3 that it (they) should be hadron-like and thus should have hadron-like fluxes and hadron-like structure-functions. This class of inelastic processes are often called明国 "diffractive scattering processes", because the same kind of "colorless object" also plays a significant role in elastic hadron-hadron scattering, and the differential cross-section data of the latter exhibit diffraction patterns similar to those observed in Optics.

What are such "colorless objects"? Can their occurrence, their properties, and their effects be understood in terms of QCD? What is the relationship between such "colorless objects" and inelastic diffraction in Optics? Can optical-geometrical concepts and methods be used to describe inelastic diffractive scattering processes at large values of invariant momentum transfer, $|t| \geq 0.2 \mathrm{GeV}^{2}$, say? In order to answer these questions, it seems useful 
to recall and/or to note the following:

(A) A number of experimental facts 99 and theoretical arguments 14 suggest that the "colorless objects" which the beam-particles encounter in LRG events are color-singlet gluonclusters.

(B) The characteristic properties of the gluons - especially the local gluon-gluon coupling prescribed by the QCD-Lagrangian, the confinement, and the non-conservation of gluonnumbers - strongly suggest that systems of interacting soft gluons are open, dynamical, complex systems with many degrees of freedom, and that such systems are in general far from equilibrium. This means in particular that, since soft gluons can be emitted and/or absorbed at any time, and everywhere in such a system, it is neither meaningful nor possible to specify the number of gluons or the amount of energy associated with the system.

(C) It has been pointed out by Bak, Tang and Wiesenfeld (BTW) 15 that a wide class of open complex systems evolve into self-organized critical (SOC) states; and local perturbations of such critical states may propagate like avalanches caused by domino effects over all length scales. Such a long-range correlation effect eventually terminates after a total time $T$, having reached a final amount of dissipative energy, and having effected a total spatial extension $S$. The quantity $S$ is called by BTW "the lifetime" of the "avalanche" and/or the "clusters". It is observed 15,16 that there are many such open dynamical complex systems in the macroscopic world, and that the distributions $D_{S}$ of $S$, and the distribution $D_{T}$ of $T$ of such BTW-avalanches/clusters obey power laws: $D_{S}(S) \propto S^{-\mu}$, and $D_{T}(T) \propto T^{-\nu}$, where $\mu$ and $\nu$ are positive real constants. Such characteristic behaviors are known 15,16 as "the fingerprints" of SOC. Having this, and the characteristic features of the gluons mentioned in (B), in mind, we are naturally led to the question: Can SOC and thus BTW-avalanches also exist in microscopic systems - at the level of quarks and gluons? Can SOC be the dynamical origin of color-singlet gluon-clusters which play the dominating role in inelastic diffractive scattering processes?

(D) In order to answer these questions we performed a systematic analysis 17 of the data for diffractive DIS where we made use of the following: (i) For a color-singlet gluon-cluster 
$c_{0}^{\star}$ to be a BTW-avalanche, its spatial size $S$ has to be directly proportional to the dissipative energy, and the latter is proportional to $x_{P}$ which is the energy fraction carried by $c_{0}^{\star}$. Hence $D_{S}(S)$ is expected to be related to the $x_{P}$-distribution of the $c_{0}^{\star}$ 's in LRG events of DIS. (ii) In such events, $x_{P}$ and the "diffractive structure function" $F_{2}^{D(3)}$ are measurable quantities. This, together with the observation that the impulse-approximation is applicable to $c_{0}^{\star}$ 's (Since the interactions between the struck $c_{0}^{\star}$ and any other neighboring color-singlets are expected to be similar to those between hadrons, the former can also be considered as Van der Waal's type of interactions.), implies that $D_{S}(S)$ can be extracted in a similar manner as the $x_{B}$-distribution of valence quarks in normal events. Furthermore, if the $c_{0}^{\star}$ 's are indeed due to SOC, there should be $c_{0}^{\star}$ 's of all lifetimes (T's). This means, for a given interactiontime $\tau_{\text {int }}$, there are always $c_{0}^{\star}$ 's whose $T$ 's are longer than $\tau_{\text {int }}$. (iii) The interaction-time $\tau_{\text {int }}$ can be estimated by making use of the uncertainty principle; to be more precise (cf. Eq.(10) of Ref.[17]), for fixed $|\vec{P}|$ and $Q^{2}$ values, $\tau_{\text {int }} \propto x_{B}$ for $x_{B} \ll 1$. Hence, in the small- $x_{B}$ region, information about the lifetime-distribution can be obtained by examining the $x_{B}$-dependence of $F_{2}^{D(3)}$. The results of the analysis can be summarized as follows: The size- and the lifetimedistributions are indeed of the form $D_{S}(S) \propto S^{-\mu}$ and $D_{T}(T) \propto T^{-\nu}$ where $\mu \approx \nu \approx 2$. Furthermore, for obvious reasons, $D_{S}(S)$ and $D_{T}(T)$ are expected to retain their powerlaw behaviors with exactly the same exponent in all Lorenz-frames. Comparisons between these results and those obtained for inelastic diffractive $\gamma p^{1}, p p^{10}$, and $\bar{p} p$ 国 processes have also been madel 7 , and the following picture emerges: In an inelastic diffractive scattering process off proton the beam-particle encounters one of the color-singlet gluon-clusters which in general exist partly inside and partly outside the confinement region of the proton. The size- and the lifetime-distributions of the clusters exhibit universal power-law behaviors, which imply in particular that such gluon-clusters are not hadron-like in the sense that they have neither a typical size, nor a typical lifetime. Furthermore, the fact 1 that the datal cannot accommodate the simple factorization assumption 22 in which a universal pomeronflux with a unique hadron-like pomeron structure function exist, gives further support to the proposed SOC-picture because a BTW-cluster cannot have a universal static structure. With 
these characteristic properties of the colorless objects in mind, we see 13 an overlap between the SOC-picture and the partonic picture for Pomeron and/or Pomeron and Reggeon 12.13 in which, beside the Pomeron, exchange of (in general an infinite number of) subleading Reggetrajectories are possible. In fact, it has been reported that very good agreement can be achieved between the datal and this kind of models. Hence, in order to differentiate between the two approaches, it is not only useful but also necessary to examine the corresponding predictions for the dependence on the invariant momentum-transfer $t$.

(E) In Optics, Frauenhofer diffraction can be observed when the wavelength of the light is less than the linear dimension of the scatterer, and when the light-source and the detecting screen are very far from the scatterer. The incident light-rays are considered as plane waves (wave vector $\vec{k}$, frequency $\omega \equiv|\vec{k}|$ ). After the scattering, the field originating from the scatterer can be written as the product of $R^{-1} \exp \left(i\left|\overrightarrow{k^{\prime}}\right| R\right)$ and the scattering amplitude $f\left(\vec{k}, \overrightarrow{k^{\prime}}\right)$. Here, $\overrightarrow{k^{\prime}}$ is the wave vector of the scattered light in the direction of observation, $\omega^{\prime} \equiv\left|\overrightarrow{k^{\prime}}\right|$ is the corresponding frequency, $R$ is the distance between the scatterer and the observation point. By choosing a coordinate system in which the $z$-axis coincides with $\vec{k}$, $f\left(\vec{k}, \overrightarrow{k^{\prime}}\right)$ can be expressed 18 as

$f(\vec{q})=(2 \pi)^{-2} \iint_{\Sigma} d^{2} \vec{b} \alpha(\vec{b}) e^{-i \vec{q} \cdot \vec{b}}$

Here, $\vec{q} \equiv \overrightarrow{k^{\prime}}-\vec{k}$ determines the change in wave vector due to diffraction; $\vec{b}$ is the impact parameter, $\alpha(\vec{b})$ is the corresponding amplitude in the two-dimensional $\vec{b}$-space (here, the $x y$-plane), and the integration extends over the region $\Sigma$ in which $\alpha(\vec{b})$ is different from zero. It is well-known that elastic scattering at small angles can be deduced 18 from this equation under the additional condition $\left|\vec{k}^{\prime}\right|=|\vec{k}|=\omega^{\prime}=\omega$, where geometry dictates that $\vec{q}$ is approximately perpendicular to $\vec{k}$ and to $\vec{k}^{\prime}$. In such cases, $\vec{q} \approx \vec{q}_{\perp}$ where $\vec{q}_{\perp}$ stands for its projection on the $x y$-plane of the chosen coordinate system. We note, the general case, in which $\vec{k}^{\prime} \neq \vec{k}$ and $\omega^{\prime} \neq \omega$, corresponds to inelastic scattering. for which we can write

$f_{\text {inel }}\left(\vec{q}_{\perp}\right)=(2 \pi)^{-2} \iint_{\Sigma} d^{2} \vec{b} \alpha_{\text {inel }}(\vec{b}) e^{-i \vec{q}_{\perp} \cdot \vec{b}}$ 
Here both $\alpha_{\text {inel }}(\vec{b})$ and $f_{\text {inel }}\left(\vec{q}_{\perp}\right)$ in general depend on the transfer of energy, and/or longitudinal momentum. Furthermore, if the scatterer is symmetric with respect to the scattering axis, Eq.(22) can also be expressed by using an integral representation for $J_{0}$, as

$f_{\text {inel }}\left(q_{\perp}\right)=(2 \pi)^{-1} \int_{0}^{\infty} b d b \alpha_{\text {inel }}(b) J_{0}\left(q_{\perp} b\right)$

where $q_{\perp}$ and $b$ stand for $\left|\overrightarrow{q_{\perp}}\right|$ and $|\vec{b}|$ respectively.

Keeping the facts mentioned in (A) - (E) in mind, we now examine the scatterer quantitatively in the rest frame of the proton target. We choose a right-handed Cartesian coordinate system with its origin $O$ at the center of the proton and the $z$-axis in the direction of the incident beam-particle which is considered point-like as it goes through the $x y$-plane at point $B \equiv(0, b)$. That is, the incident beam and the center $O$ of the proton determine the scattering plane (yz-plane) of the collision event, where the distance $\overline{O B}$ is the corresponding impact parameter $b$. Since we are dealing with inelastic scattering (where the momentum transfer also in the longitudinal direction can be large) it is possible to envisage that the scattering takes place at one point in space, and effectively 19 at the point $B$, where it meets colorless gluon-clusters. The latter are BTW-avalanches due to SOC initiated by local interactions in systems of soft gluons. Since gluons carry color, the interactions which lead to the formation of gluon-clusters must take place inside the confinement region of the proton. This means, while a considerable part of the created color-singlet clusters can be outside the proton, the location $A$ where such an avalanche is initiated must be inside the proton. That is, in terms of $\overline{O A} \equiv r, \overline{A B} \equiv R_{A}(b)$, and proton's radius $r_{p}$, we have $r \leq r_{p}$ and $\left[R_{A}(b)\right]^{2}=b^{2}+r^{2}-2 b r \cos \angle B O A$. For a given impact parameter $b$, the relevant quantity is the mean distance $\left\langle R_{A}^{2}(b)\right\rangle^{1 / 2}=\left(b^{2}+a^{2}\right)^{1 / 2}, a^{2} \equiv 3 / 5 r_{p}^{2}$, which is obtained by averaging over all allowed locations of $A$ in the confinement region. That is, we can model the effect of confinement in avalanche-formation by picturing that all such BTW-avalanches in particular those which contribute to scattering events characterized by a given $b$ are initiated from an "effective initial point" $\left\langle A_{b}\right\rangle$. Note also that since an avalanche is a dynamical object, it in general expands and propagates within its lifetime (in any one of the $4 \pi$ directions away 
from $\left.\left\langle A_{b}\right\rangle\right)$. It is envisaged that the expansion and propagation of such an avalanche cannot proceed without limits. This is not only because the gluon-density is expected to decrease with increasing distance from $O$, but also because color-forces between the constituents of $c_{0}^{\star}$ increase (consistent with confining potentials) when their distance become larger. That is, those constituents "going too far" will be "pulled back" to the proton's confinement region.

An explicit expression for the amplitude $\alpha_{\text {inel }}(b)$ in Eq.(3), can be readily written down by taking the following into account: $(\alpha)$ SOC dictates that there are avalanches of all sizes $\left(S_{i}\right.$ 's) and that the number-density of avalanches of $S_{i}$ is $D_{S}\left(S_{i}\right) \propto S_{i}^{-\mu}$ where experiments show 1 17 $\mu \approx 2$. ( $\beta$ ) Since the interactions between the struck $c_{0}^{\star}$ and any other color-singlets should be of Van der Waals type, it can simply be "carried away" by the beam-particle. Simple geometrical considerations suggest that the chance for an avalanche of size (i.e. volume) $S_{i}$ to be hit (on the plane perpendicular to the incident axis) should be proportional to $S_{i}^{2 / 3}$. $(\gamma)$ Since for a given $b$, the distance in space between $\left\langle A_{b}\right\rangle$ and $B \equiv(0, b)$ is $\left(b^{2}+a^{2}\right)^{1 / 2}$, the number of avalanches which pass a unit area on the shell of radius $\left(b^{2}+a^{2}\right)^{1 / 2}$ centered at $\left\langle A_{b}\right\rangle$ is proportional to $\left(b^{2}+a^{2}\right)^{-1}$, provided that (due to causality) the lifetimes ( $T$ 's) of these avalanches are not shorter than $\tau_{\min }(b)$. The latter is the time interval for an avalanche to travel (with constant velocity, say) from $\left\langle A_{b}\right\rangle$ to $B$ that is $\tau_{\min }(b) \propto\left(b^{2}+a^{2}\right)^{1 / 2}$, and only those avalanches having lifetimes $T \geq \tau_{\min }(b)$ can contribute to such an event. Now, since avalanches are due to SOC and the chance for an avalanche of lifetime $T$ to exist is $D_{T}(T) \propto T^{-\nu}$ where而 $\nu \approx 2$, the proper fraction can be obtained by integrating $T^{-2}$ over $T$ from $\tau_{\min }(b)$ to infinity, which is $\left(b^{2}+a^{2}\right)^{-1 / 2}$.

Hence, for inelastic diffractive scattering processes in which the beam-particles encounter avalanches of size $S_{i}$, the amplitude which we denote by $\alpha_{\text {inel }}\left(b \mid S_{i}\right)$ can be obtained from the square-root of $D_{S}\left(S_{i}\right)$ mentioned in $(\alpha)$, and by taking the weighting factors mentioned in $(\beta)$ and $(\gamma)$ into account. The result is:

$\alpha_{\text {inel }}\left(b \mid S_{i}\right)=$ const. $S_{i}^{-1 / 3}\left(b^{2}+a^{2}\right)^{-3 / 2}$.

By inserting this in Eq.(33), we obtain the corresponding amplitude in $\vec{q}_{\perp}$-space: $f_{\text {inel }}\left(q_{\perp} \mid S_{i}\right)$. 
Here, $q_{\perp}=\left|\vec{q}_{\perp}\right| \approx \sqrt{|t|}$ is the corresponding momentum-transfer. The integration can be carried out analytically 21, and the result (after the summation over $i$ ) for the inclusive inelastic single diffractive differential cross-section $d \sigma / d t$ is

$d \sigma / d t=C \exp (-2 a \sqrt{|t|})$

Here $a^{2} \equiv \frac{3}{5} r_{p}^{2}$, and $C$ is an unknown constant. Note that the value for $a$ is the same for different incident energies, and for different projectiles: $\gamma^{\star}, \gamma, p, \bar{p}$, etc.

To compare this result with experiments, we calculate $a$ by using its definition $a^{2} \equiv \frac{3}{5} r_{p}^{2}$ and the experimental value22 for $r_{p}$ which is $0.81 \mathrm{fm}$. We note, while "the slope" $2 a$ depends only on the radius of the target-hadron, the normalization constant $C$ is expected to be different for different projectiles. The comparisons are shown in Figs.1 and 2.

A few remarks should be made in this connection: (i) The $d \sigma / d t$-data for $p p, \bar{p} p, \gamma p$ and $\gamma^{\star} p$ reactions are consistent with no energy $(\sqrt{s}$ and/or $W)$-dependence. In particular, in contrast to elastic $p p$-scattering, there is no indication of "shrinkage". (ii) The figures show: In a limited range of $\sqrt{|t|}$, curves with very much different $t$-behaviors can yield rather similar results. (iii) The existing data show that the coefficients of $\sqrt{|t|}, t$, or $t^{2}$ in the curves for $d \sigma / d t$ are consistent with no $M_{x}$-dependence. This is in particular the case for $\gamma p$-reactions (see in this connection the second paper of Ref.[9]). (iv) A simple analytic expression for $d^{2} \sigma / d t d x_{P}$ can be readily obtained by taking $S_{i} \propto x_{P}$ into account in Eq.(4) and by integrating over $b$. The result which is shown to be in good agreement with the existing data for $p p$ - and $\bar{p} p$-reactions will be presented in Ref.[20]. Evidently, further experiments, especially measurements of $d \sigma / d t$ and $d^{2} \sigma / d t d\left(M_{x}^{2} / W^{2}\right)$ for $\gamma p \rightarrow X p$ at larger $|t|$-values would be helpful.

We thank T. T. Chou for correspondence, K. Tabelow and W. Zhu for discussions, and FNK der FU Berlin for financial support. Y. Zhang thanks Alexander von Humboldt Stiftung for the fellowship granted to him. 


\section{FIGURES}

Fig. 1. The $d \sigma / d t$ data taken from Refs. [10, [1] are plotted against $\sqrt{|t|}$ in the measured kinematical range. The solid line is our result. The dashed and dot-dashed lines show the conventional fits $d \sigma / d t \propto \exp \left[B t+C t^{2}\right]$. The former is the UA4-fit to their data [11] with $B=8.0 \pm 0.1 \mathrm{GeV}^{-2}$ and $C=2.3 \pm 0.1 \mathrm{GeV}^{-4}$. The latter is a fit to the same expression for all the data points in this figure, where $B=5.7 \pm 0.1 \mathrm{GeV}^{-2}$ and $C=0.8 \pm 0.1 \mathrm{GeV}^{-4}$.

Fig. 2. The $d \sigma / d|t|$ data from Refs. [0],9] for $\gamma^{\star}$ and $\gamma$ induced reactions are plotted against $\sqrt{|t|}$. They are shown as circles and squares respectively. Here, the empty circles and squares are the data from (the transparencies of) Ref. [4] while the solid ones are those from Refs. [9]. The solid lines stand for our result. The dashed and dotted lines are fits given in Refs. [0].9]: $d \sigma / d t \propto \exp (-b|t|)$, where $b=7.3 \pm 0.9 \pm 1.0 \mathrm{GeV}^{-2}$ and $b=6.8 \pm 0.9+1.2 /-1.1 \mathrm{GeV}^{-2}$. 


\section{REFERENCES}

1. W. Friedrich, P. Knipping and M. Laue, Ann. d. Phys. 41, 971 (1913).

2. See e.g. G. Bellettini et al., Nucl. Phys. 79, 609 (1966).

3. See e.g. J. Orear, Physik, Carl Hanser Verlag München Wien (1979), p. 507.

4. See, e.g. R. J. Glauber, in Lectures in Theoretical Physics, ed. by W. E. Brittin and L. G. Dunham, Interscience publishers, Inc., N. Y. (1959), p. 315.; R. Serber, Rev. Mod. Phys., 649 (1964); N. Byers and C. N. Yang, Phys. Rev. 142, 976 (1966); T. T. Chou and C. N. Yang, Phys. Rev. 170, 1591 (1968); 175, 1832 (1968); D 22, 610 (1980); U. Amaldi, M. Jacob, and G. Matthiae, Ann. Rev. Nucl. Sci. 26, 385 (1976); M. M. Block and R. N. Cahn, Rev. Mod. Phys. 57, 563 (1985).

5. M. Derrick et al., Phys. Lett. B 315, 481(1993); T. Ahmed et al., Nucl. Phys. B 429, $477(1994)$.

6. See e. g. M. Derrick et al., ZEUS Coll., Phys. Lett. B345, 576(1995); S. Aid et al., H1 Coll., Phys. Lett. B354, 494(1995); and the papers cited therein.

7. See e.g. E. Gallo, in Proc. of the 18th Int. Symp. on Lepton - Photon Interactions, edited by A. De Roeck and A. Wagner, Singapore, World Scientific (1998).

8. T. Ahmed et al., Phys. Lett. B 348, 681(1995); M. Derrick et al., Z. Phys. C 68, 569(1995); ibid, C 70, 391(1996), C. Adloff et al.,Z. Phys. C76, 613 (1997), J. Breitweg et al., Eur. Phys. J. C6, 43 (1999).

9. J. Breitweg et al., Eur. Phys. J. C1, 81-96 (1998); Eur. Phys. J. C2, 237-246 (1998).

10. M.G. Albrow et al., Nucl. Phys. B 108, 1 (1976).

11. D. Bernard et al., Phys. Lett. B 186, 227 (1987); A. Brandt et al., preprint hepph/9710004.

12. See e.g. G. Ingelman, K.Prytz, Z. Phys. C58, 285 (1993). 
13. G. Sterman, private communication.

14. See e.g. F. E. Low, Phys. Rev. D12, 163 (1975); S. Nussinov, Phys. Rev. Lett. 34, 1286 (1975); C. Boros, Z. Liang and T. Meng, Phys. Rev. D54, 6658 (1996).

15. P. Bak, C. Tang and K. Wiesenfeld, Phys. Rev. Lett. 59, 381(1987); Phys. Rev. A 38, $364(1988)$.

16. See e.g. P. Bak, "How nature works" Spinger-Verlag, NY (1996).

17. C. Boros, T. Meng, R. Rittel and Y. Zhang, preprint hep-ph/9704285.

18. See e.g. L. D. Landau and E. M. Lifshitz, The Classical Theory of Fields, (Second revised addition), Pergamon Press, Oxford (1962), p. 177 and p. 165.

19. This is the point from where the main contribution comes. Contributions from points outside the $x y$-plane, and effects caused by the environment on the propagating avalanche, can be, and have been, taken into account in a more elaborated modeling procedure 20. The final result turns out to be the same.

20. T. Meng, R. Rittel, K. Tabelow and Y. Zhang, in preparation.

21. I. S. Gradshteyn and I. M. Ryzhik, Table of Integrals, Series and Products, Academic Press, NY (1980), p. 682.

22. See e.g. F. Halzen and A. D. Martin, Quarks and Leptons, John Wilsey \& Sons, NY (1984), p. 179 . 


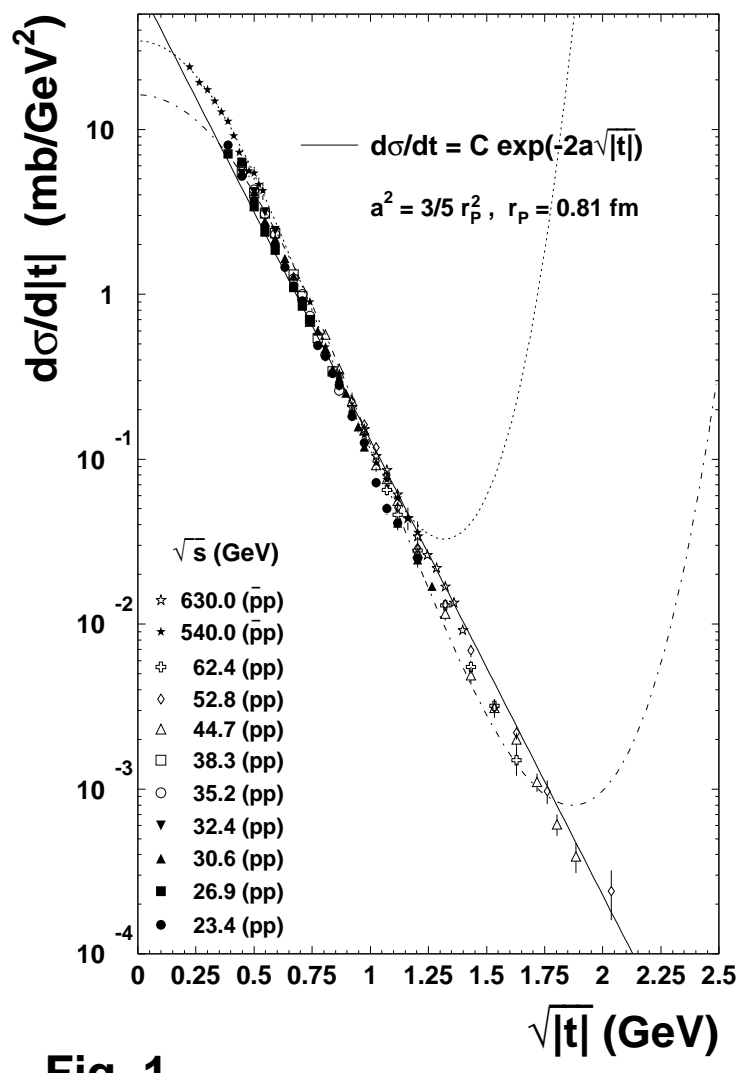

Fig. 1
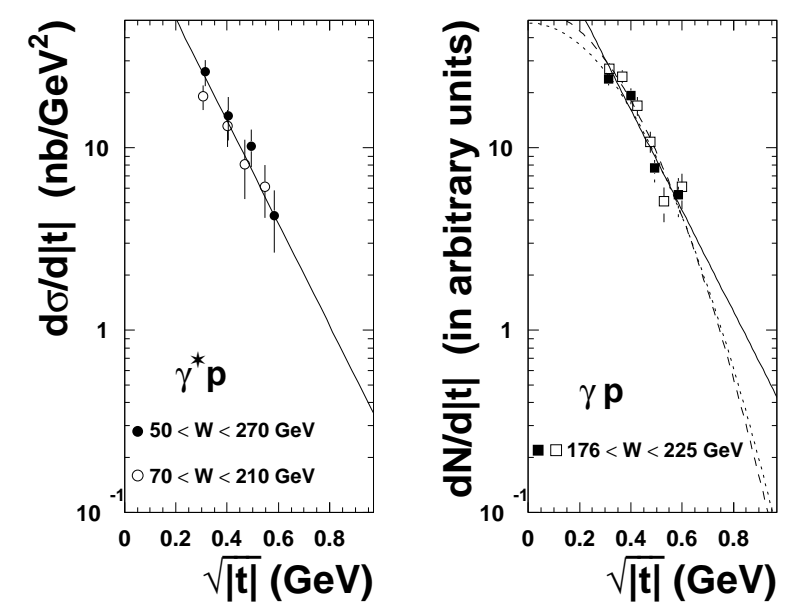

Fig. 2 